\title{
Analyzing the Sharkskin Instability
}

\section{The stretching and recoiling of polymer chains leads to the characteristic} ridge pattern as a soft material exits a narrow nozzle.

\section{By Rachel Berkowitz}

W hen a polymer melt squeezes through a small nozzle-a process common to all plastics manufacturing-the extruded material's surface becomes roughened because of a phenomenon known as the sharkskin instability. The polymer-processing community has long known that these scaly ridges are caused by large tensile stresses in the melt near the nozzle, but simulations have so far failed to capture the underlying physical mechanism in detail. Stylianos Varchanis of the University of Patras, Greece, and his colleagues now report a simple physical model that reproduces the flow's transition from smooth to scaly [1].

Based on continuum mechanics principles, the team numerically simulated a two-dimensional polymer melt as it was squeezed through a wide, low slit. By tracing the paths of discrete fluid parcels, they observed that the extruded melt was subject to tension as it passed near the edge of the slit. This tension relaxed as the melt moved away from the slit. In a low-viscosity melt, the stretched polymer chains relaxed uniformly, leaving the surface of the flow smooth. In a

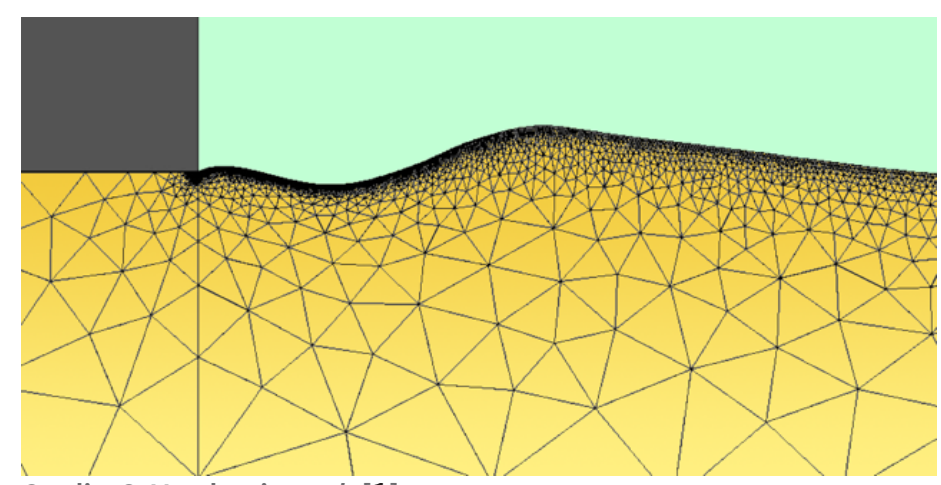

Credit: S. Varchanis et al. [1]
Video 1: Tension in the flow as the melt extrudes near the edge of the slit stretches the polymer chains. When the polymer chains recoil, they can create the flow instability that is responsible for the sharkskin texture.

Credit: S. Varchanis et al. [1].

high-viscosity melt, the polymer chains recoiled sharply, bunching together and setting up traveling waves-the familiar perpendicular ridges-that propagated along the surface of the melt from the slit toward the flow front.

Knowing the mechanism behind the sharkskin instability could lead to more precise control over polymer-processing conditions, helping to eliminate the effect and allowing increased flow rates without sacrificing product quality. The researchers say that the work will also help to advance fundamental understanding of the relation between the microscopic conformations of polymer chains and macroscopically observed phenomena.

Rachel Berkowitz is a Corresponding Editor for Physics based in Vancouver, Canada.

\section{REFERENCES}

1. S. Varchanis et al., "Origin of the sharkskin instability: Nonlinear dynamics," Phys. Rev. Lett. 127, 088001 (2021). 